\title{
Static and Dynamic Longitudinal Structural Analyses of Cognitive Changes in Old Age
}

\author{
Paolo Ghisletta ${ }^{a, b, c}$ Ulman Lindenberger ${ }^{d}$ \\ a Center for Interdisciplinary Gerontology and ${ }^{b}$ Faculty of Psychology and Educational Sciences, University of \\ Geneva, Geneva, and ${ }^{\circ}$ Center for Life Course and Life Style Study, Universities of Lausanne and Geneva, \\ Switzerland; dDepartment of Psychology, Saarland University, Saarbrücken, Germany
}

\section{Key Words}

Latent growth and multilevel models · Cognitive aging

\begin{abstract}
Background: Among the main data-analytical advances of recent decades are Latent Growth Models (LGM) and Multilevel Models (MLM) for the analysis of longitudinal data. Objective: We discuss the relative advantages and disadvantages of the two analytical methods and offer some practical guidelines concerning the choice between LGM and MLM based on (a) completeness and balance of the data, (b) theoretical functional form of change examined, (c) examination of the error structure, (d) theoretical relations among differential level effects and differential change effects, and (e) role of timeinvariant covariates. Methods: To discuss LGM and MLM, we provide illustrations from applications to the Berlin Aging Study (BASE) and the Swiss Interdisciplinary Longitudinal Study on the Oldest Old (SWILSO-O). Results: As predicted by two-component theories of lifespan cognition, performance on a vocabulary test (indicator of broad crystallized intelligence) did not decline over time, while scores on a digit letter test (indicator of broad fluid intelligence) decreased over 6 years. Differential level effects were obtained on both variables,
\end{abstract}

\section{KARGER}

Fax +41613061234 E-Mail karger@karger.ch www. karger.com
(C) 2004 S. Karger AG, Basel

0304-324X/04/0501-0012\$21.00/0

Accessible online at: www. karger.com/ger while average and differential change effects were obtained only for the digit letter test. In a second set of analyses, we tested the error-free effect that a broad fluid intelligence indicator exerted on the latent yearly change in a broad crystallized intelligence indicator, and vice versa. In both data sets we obtained strong evidence for a more reliable effect of the fluid indicator on the change in the crystallized indicator. This evidence provided support for the dedifferentiation hypothesis of cognitive abilities in very old age. Conclusions: New insights into cognitive aging phenomena can be gained with proper applications of LGM and MLM. We posit that the choice between LGM and MLM and their specification rests on theoretical and empirical motives to be defined a priori.

Copyright $@ 2004$ S. Karger AG, Basel

During recent decades, lifespan developmental psychologists have witnessed some progress from conceptual, theoretical, methodological, and data-analytical perspectives $[1,2]$. Among newer statistical models are Latent Growth Models (LGM) and Multilevel Models (MLM) for the analysis of longitudinal data. In this paper we discuss some applications of analytical models to the study of cognitive aging. To elucidate the themes discussed, we provide two empirical examples from our research.
Paolo Ghisletta

Center for Interdisciplinary Gerontology, University of Geneva

Route de Mon-Idée 59

CH-1226 Thônex (Switzerland)

Tel. +41 2230566 04, Fax +41 2234890 77, E-Mail paolo.ghisletta@cig.unige.ch 
Table 1. Description of variables from the BASE and SWILSO-O longitudinal designs

\begin{tabular}{|c|c|c|c|c|c|c|c|c|c|c|}
\hline \multirow[t]{2}{*}{ Cognitive domain } & \multicolumn{7}{|c|}{$\begin{array}{l}\text { BASE } \\
\text { occasion of measurement }\end{array}$} & \multicolumn{3}{|c|}{$\begin{array}{l}\text { SWILSO-O } \\
\text { occasion of measurement }\end{array}$} \\
\hline & $\mathrm{IA}_{1}$ & $\mathrm{IP}_{1}$ & $\mathrm{IA}_{2}$ & $\mathrm{IA}_{3}$ & $\mathrm{IP}_{3}$ & $\mathrm{IA}_{4}$ & $\mathrm{IP}_{4}$ & 1 & 2 & 3 \\
\hline Broad Gf/mechanic & DL & DL, IP & DL & DL & DL, IP & DL & DL, IP & $\mathrm{CO}$ & $\mathrm{CO}$ & $\mathrm{CO}$ \\
\hline Broad Gc/pragmatic & - & VO, SW & - & - & VO, SW & - & VO, SW & $\mathrm{CA}$ & $\mathrm{CA}$ & $\mathrm{CA}$ \\
\hline Time in study, years & 0.00 & 0.13 & 1.95 & 3.76 & 3.99 & 5.55 & 6.03 & 0.00 & 1.35 & 2.31 \\
\hline $\mathrm{n}$ & 516 & 516 & 361 & 244 & 208 & 164 & 132 & 376 & 289 & 247 \\
\hline
\end{tabular}

IA = Intake assessment; IP = intensive protocol; $\mathrm{DL}=$ digit letter; $\mathrm{IP}=$ identical pictures; $\mathrm{VO}=$ vocabulary; $\mathrm{SW}=$ spot-a-word; $\mathrm{CO}=\mathrm{cross}$ out; $\mathrm{CA}=$ category .

\section{Latent Growth Models and Multilevel Models}

LGM appeared in developmental psychology less than two decades ago [3]. MLM (highly similar to random effect, mixed, and hierarchical models) applied to longitudinal analyses developed independently [4, 5]. Today, both methodologies are commonly applied in the field of cognitive aging [e.g., 6-8]. The similarities and differences of LGM and MLM, although documented in the literature [e.g., 9-12], are not always clear and the empirical researcher is sometimes left wondering when to prefer one approach over the other. In short, MLM may be favored over LGM when: (a) the data are highly incomplete and/or unbalanced; (b) the functional form of change can be represented by a common mathematical function (e.g., polynomial, logistic, exponential); (c) the error structure can be represented by a common matrix (e.g., Toeplitz, independent-diagonal, auto-regressive, unstructured); (d) the theory examined does not mandate relations other than covariances among change components of different variables (i.e., static condition), and (e) the time-invariant covariates act uniquely as predictors of differential level and change effects. LGM may be favored over MLM when: (a) the degree of data incompleteness and/or unbalance is limited; (b) the change function is to be represented by more complex mathematical formulations or is to be estimated by the data; (c) the error structure is of theoretical interest, requiring flexible modeling; (d) the relations among the variables' level and change components cannot be represented by covariances (e.g., dynamic condition), and (e) the time-invariant covariates assume theoretical roles other than predictors of differential level and change effects. These conditions, however, can be adjusted and at times overwritten given sufficient familiarity with the analytical method chosen and the software utilized for their implementation.

To illustrate the notions mentioned above, we discuss applications of LGM and MLM to the Berlin Aging Study (BASE) [13] and the Swiss Interdisciplinary Longitudinal Study on the Oldest Old (SWILSO-O) [14].

\section{Description of Research Studies}

BASE is a multidisciplinary study of aging combining psychology, sociology and social policy, internal medicine and geriatrics, and psychiatry. At its onset (in 1989) it consisted of 516 individuals randomly sampled from the city registry of Berlin, Germany, stratified by gender and age (six groups of 5 years each from age 70 ; mean age $=$ 84.9 years, age range $=69.7-103.1$ years) $[\mathrm{cf} .15$ ] . The study is now in its fifth wave, but only data of the first four occasions are presented here. The first, third, and fourth waves of data collection were preceded by an initial assessment, during which fewer variables were administered. The full longitudinal cognitive battery included eight variables. However, for simplicity and illustration purpose, we will limit the analyses presented here to two markers of perceptual speed (digit letter and identical pictures) and two markers of verbal knowledge (vocabulary and spot-a-word). The data unbalance is apparent from table 1. In particular, the digit letter test was assessed seven times, while the other selected cognitive variables were limited to three assessments.

SWILSO-O is an interdisciplinary study of aging involving sociology, social psychology, cognitive psychology, social medicine, and econometrics. Two age-parallel cohorts comprise the sample, the first begun in 1994 (now 
in its eighth wave), the second in 1999 (currently in its fourth wave). The cognitive tests illustrated here were included only in the latter cohort. At inception, the second cohort comprised 376 participants stratified by geographical region (191 in rural Valais, Switzerland, vs. 185 in urban Geneva, Switzerland). The mean age at time 1 was 81.9 years, the age range $79.5-84.5$ years. In contrast to the BASE cognitive design, the SWILSO-O cognitive design, albeit less comprehensive in its research pursuits, is balanced. Because of the shorter duration and the different age composition of the sample, the dropout rate at the end of the periods considered is smaller for SWILSO$O$ than for BASE $(34.31 \%$ dropout rate in 2.31 years for SWILSO-O versus $74.42 \%$ throughout 6.03 years for BASE).

\section{Static Relations between Broad Gf/Mechanic and Broad Gc/Pragmatic Markers}

In this first set of analyses we were mainly interested in relations among cognitive variables of different domains, as hypothesized by two component theories of lifespan cognition [e.g., 16]. In particular, we were interested in the relationships among markers of broad $\mathrm{Gf} /$ mechanic and broad Gc/pragmatic domains. For didactical reasons, we will illustrate this application only with the digit letter (assessed seven times) and the vocabulary (assessed three times) tests of BASE. Classical model of change would have required that the variables analyzed be balanced, that is to say assessed an equal number of times. This inevitably would have resulted in the loss of more than half of the digit letter assessments. More modern models can not only accommodate incomplete data (unavoidable in longitudinal aging studies), but also unbalanced data.

The theoretical motivation of the analyses could be addressed with covariance relations among the level and change components of each variable. Moreover, we limited ourselves to polynomial change, and were not substantively motivated to investigate the structure of the residual effects of change. Hence, we favored the MLM approach. We defined change as the passing of time in years since the beginning of the study (i.e., we applied the time in study temporal definition because theoretical motives did not favor a different temporal definition). To obtain unbiased estimates of person-level covariances we centered the data around each person's mean of time in study [cf. 17-19].

We first studied the change function of each variable in a univariate fashion. This implied two separate, unrelated
MLM. For each variable we studied the existence (i.e., significant difference from 0 ) of the random effect of the intercept (analogous to the variance of the level factor in LGM), fixed linear effect of time (i.e., mean of the linear slope factor), the random linear effect of time (i.e., variance of the linear slope factor), and the 'level 2' covariance (i.e., covariance between level and linear slope factors over participants). For the digit letter variable, assessed up to seven times, we also tested for fixed and random quadratic effects of time (i.e., for the mean and the variance of a quadratic slope factor) and these were found not to be statistically significant.

The two cognitive variables displayed reliable fixed and random intercept effects, while only digit letter displayed both reliable fixed and random linear time effects. Hence, on average, the sample's performance varied around the intercept of both the digit letter and vocabulary tests. However, average and differential linear change were displayed only on the digit letter test. The sample's performance on the vocabulary test stayed constant during the period considered. This situation (variation around the intercept of both tests and only variation around the linear slope of digit letter) gave rise to a three by three 'level 2' covariance matrix, that is a covariance matrix about the participants' heterogeneity around the intercepts and linear slope components. The covariance matrix contained the information about how much the sample varied on the intercept of both tests, how much the sample varied with the respect to the change in digit letter, and about the covariations among these components. Statistical testing of this covariance matrix concluded that all six elements were different from zero. The level variance was 90.75 for digit letter and 74.73 for vocabulary, while the slope variance of digit letter was 0.78 . The correlation between the levels of digit letter and vocabulary was 0.64 . The level and slope of digit letter correlated 0.49 , while the slope of digit letter correlated 0.28 with the level of vocabulary.

As predicted by two-component theories of lifespan cognition, the broad Gf/mechanic marker displayed negative age changes within the present longitudinal observation interval of up to 6 years, while the broad Gc/pragmatic markers (e.g., vocabulary) did not [cf. 7]. Furthermore, the positive manifold phenomenon usually observed in cross-sectional studies among different cognitive abilities was here observed in latent space as a high correlation between the levels of both markers. The application of MLM allowed for the estimation of longitudinal age gradients of the broad $\mathrm{Gf} /$ mechanic marker and for relations among its level and linear change components. The level 
performance on the vocabulary test is not only informative of the level performance of the digit letter test, but also of the linear change in the digit letter test. However, no statement about timing (i.e., possible longitudinal lead-lag relationships among the two cognitive tests) can be inferred by inspecting the static level 2 covariance matrix

\section{Dynamic Relations between Broad Gf/Mechanic and Broad Gc/Pragmatic Markers}

The second set of analyses [see also 20] was concerned with the testing of the dedifferentiation hypothesis of cognitive abilities in very old age, according to which performance on cognitive abilities is more generalized and less specific for older individuals, probably due to biological changes occurring at the brain level [16, 21, see also 22]. An untested assumption of the dedifferentiation hypothesis is that the broad Gf/mechanic markers affect directly the negative changes of broad Gc/pragmatic markers in old age.

The phenomenon investigated is hypothesized to occur over chronological age, so the most sensible definition of time is in terms of chronological age. The theoretical paradigm can no longer be examined with correlational relations among variables' characteristics. Rather, a sort of a cross-lagged parameter capturing the unidirectional effect exerted by one variable on the change of the other variable is needed. We hence opted for a variation of LGM, called Dual Change Score Model (DCSM) [23, 24]. The DCSM builds upon an LGM by defining the change in the variable of interest as a composite of the latent slope component and the auto-proportion effect due to the previous measurement, free of unrelated variance. In a multivariate setting the DCSM additionally defines the direct and error-free proportionality effect of one variable onto the change in the other variables. If this effect is statistically reliable, we say that the variables analyzed are linked dynamically, because the total effect of one variable on the other will change in time. The dynamic links are assessed independently of differences in reliability among the variables analyzed [20, 25].

Broad Gf/mechanic abilities were marked with a composite of the digit letter and identical pictures tests in BASE and the cross-out test in SWILSO-O, while broad Gc/pragmatic abilities with a composite of the vocabulary and spot-a-word tests in BASE and with a category (fruit) test in SWILSO-O. In each example, the effect size of the directional effect from the broad Gf/mechanic marker to the broad Gc/pragmatic marker was compared to the effect size of the opposite directional effect, after accounting for the auto-proportionality effects. In the BASE application estimating the parameter from perceptual speed to the change in verbal knowledge represented a $\chi^{2}$ decrease of 31 points for one degree of freedom, while the opposite parameter (from verbal knowledge to the change in perceptual speed) amounted to a $\chi^{2}$ drop of 10 points. The effect size of the former parameter was hence much greater. Similarly, in the SWILSO-O application estimating the parameter from perceptual speed to change in category caused a reduction of $4 \chi^{2}$ points, while estimating that from category to change in perceptual speed reduced the $\chi^{2}$ index minimally. This application provides an empirical validation based on longitudinal data of the dedifferentiation hypothesis of cognitive abilities in old and very old age, independently of the sample's age heterogeneity.

\section{Discussion}

LGM and MLM extended the repeated-measures (M)ANOVA model by focusing not only on mean trajectories, but also on variance expectations, allowing for the separation of change-related variance from residual (e.g., error, retest effects) variance. Moreover, within LGM and MLM the distinction between level and change is formalized, so that several hypotheses about change correlating with initial level can be addressed statistically. Advanced applications of LGM and MLM allow also the modeling of non-polynomial change functions and of the empirical 'optimal' change function [e.g., 8].

The DCSM is a particular structural equation model nesting and combining the LGM-MLM and the more classical auto-regressive Markov-type model. Indeed, the DCSM retains the appeals of the LGM-MLM approach and adds the estimation of the auto-proportionality parameter. In multivariate applications the DCSM becomes particularly interesting because it allows for the estimation of the dynamic effects among changing variables, after accounting for unrelated variance, differences in reliability, auto-proportionality effects, as well as for the effects of covariates.

\section{Conclusions}

Recent advances in data analytical strategies allow researchers to relax many atheoretical assumptions of the past. Nowadays theories and hypotheses of aging can be 
more adequately tested and sounder conclusions can be drawn. However, any statistical model is only useful if its theoretical motives are explicit and the match between the theoretical and the empirical levels is as satisfying as possible. LGM and MLM are but two analytical strategies available to ameliorate theory testing processes. While they are clearly not free from problems (e.g., assumptions of data missing at random, of ergodicity, and of sample homogeneity), LGM, MLM, related models of change, and, most of all, their appropriate applications can deepen our understanding of behavioral processes dear to lifespan developmentalists and gerontologists alike.

\section{References}

1 Collins LM, Horn JL: Best methods for the analysis of change. Washington, American Psychological Association, 1991.

2 Collins LM, Sayer M: New methods for the analysis of change. Washington, American Psychological Association, 2001.

3 McArdle JJ: Latent growth within behavior genetic models. Behav Genet 1986;16:163200.

4 Laird NM, Ware JH: Random-effects models for longitudinal data. Biometrics 1982;38:963974.

5 Bryk AS, Raudenbush SW: Application of hierarchical linear models to assessing change. Psychol Bull 1987;101:147-158.

6 Hofer SM, Christensen H, Mackinnon AJ, Korten AE, Jorm AF, Henderson AS, Easteal S: Change in cognitive functioning associated with ApoE genotype in a community sample of older adults. Psychol Aging 2002;17:194-208.

7 Singer T, Verhaeghen P, Ghisletta P, Lindenberger U, Baltes PB: The fate of cognition in very old age: Six-year longitudinal findings in the Berlin Aging Study (BASE). Psychol Aging 2003;18:318-331.

8 Ghisletta P, McArdle JJ: Latent growth curve analyses of the development of height. Structural Equation Modeling 2001;8:531-555.

9 McArdle JJ, Hamagami F: Multilevel models from a multiple group structural equation perspective; in Marcoulides GA, Schumaker RE (eds): Advanced Structural Equation Modeling. Issues and Techniques. Mahwah, Lawrence Erlbaum, 1996, pp 89-124.

10 Chou C-P, Bentler PM, Pentz MA: Comparisons of two statistical approaches to study growth curves: The multilevel model and latent curve analysis. Structural Equation Modeling 1998;5:247-266.
11 MacCallum RC, Kim C, Malarkey WB, Kiecolt-Glaser JK: Studying multivariate change using multilevel models and latent curve models. Multivar Behav Res 1997;32:215-253.

12 Lindenberger U, Ghisletta P: Modeling longitudinal changes in old age: From covariance structures to dynamic systems; in Dixon RA, Bäckman L, Nilsson LG (eds): New Frontiers in Cognitive Aging. Oxford, Oxford University Press, in press.

13 Baltes PB, Mayer KU: The Berlin Aging Study: Aging from 70 to 100 . New York, Cambridge University Press, 1999.

14 Lalive d'Epinay C, Pin S, Spini D: Présentation de SWILSO-O, une étude longitudinale suisse sur le grand âge: L'exemple de la dynamique de la santé fonctionnelle. Année Gérontol 2001; 15:78-96.

15 Lindenberger U, Gilberg RD, Little TD, Nuthmann R, Pötter U, Baltes PB: Sample selectivity and generalizability of the results of the Berlin Aging Study; in Baltes PB, Mayer KU (eds): The Berlin Aging Study: Aging from 70 to 100. New York, Cambridge University Press, 1999, pp 56-82.

16 Lindenberger U: Lifespan theories of cognitive development; in Smelser NJ, Baltes PB (eds): International encyclopedia of the social and behavioral sciences. Oxford, Elsevier, 2001, pp 8848-8854.

17 Wainer H: The centercept: An estimable and meaningful regression parameter. Psychol Sci 2000;11:434-436.

18 Kreft IGG, de Leeuw J, Aiken LS: The effect of different forms of centering in hierarchical linear models. Multivariate Behav Res 1995;30: $1-21$.
19 Mehta PD, West SG: Putting the individual back into individual growth curves. Psychol Methods 2000;5:23-43.

20 Ghisletta P, Lindenberger U: Age-based structural dynamics between perceptual speed and knowledge in the Berlin Aging Study: Direct evidence for ability dedifferentiation in old age. Psychol Aging, in press.

21 Baltes PB, Lindenberger U, Staudinger UM: Life-span theory in developmental psychology; in Lerner RM (ed): Handbook of Child Psychology. 1. Theoretical Models of Human development. New York, Wiley, 1998, pp 1029_ 1143.

22 Li S-C, Lindenberger U, Hommel B, Aschersleben G, Prinz W, Baltes PB: Lifespan transformations in the couplings between intellectual abilities and constituent cognitive processes. Psychol Sci, in press.

23 McArdle JJ, Hamagami F: Latent difference score structural analyses for linear dynamic analyses with incomplete longitudinal data; in Collins LM, Sayer M (eds): New Methods for the Analysis of Change. Washington, American Psychological Association, 2001, pp 137-175.

24 Hamagami F, McArdle JJ: Advanced studies of individual differences linear dynamic models for longitudinal data analysis; in Marcoulides GA, Schumacker RE (eds): New Developments and Techniques in Structural Equation Modeling. Mahwah, Lawrence Erlbaum, 2001, pp 203-246.

25 McArdle JJ, Hamagami F, Meredith W, Bradway KP: Modeling the dynamic hypotheses of Gf-Gc theory using longitudinal life-span data. Learn Indiv Differ 2000;12:53-79. 